\section{Troubleshooting Eosin Staining, And A Brighter Eosin Stain}

Gary Gill, Diagnostic Cytology Laboratories, Inc. garywgill@email.msn.com

Consider the following systematic troubleshooting recommendations for eosin staining problems, as well as for other dyes:

1) Be sure the eosin solution is working. Stain a control tissue, the type is irrelevant, in eosin only, and process routinely. Try to stain a buccal smear, rinse, and examine wet. In other words, do the least possible to the stained tissue to eliminate unexpected source of variation and have the most confidence in your evaluation. Always run a control tissue as a quality assurance measure with every new batch of stain.

2) If a ready made eosin is causing the problem, the vendor may have misformulated it. Such mistakes don't occur frequently, but they do occur. I know of several from first-hand experience as a consultant.

3) If too little stain is present, whether in the control or routine tissues, then either too little is getting in, or enough is getting in, but too much is getting out.

4) If too little eosin is getting in, possible reasons include:

a) If the stain is made from scratch, correct for dye content variation.

b) If the stain is made from scratch and the eosin powder is "old", be sure the dye content is $90 \%$ or greater. Older lots that contain between $80-89 \%$ dye content sometimes contained unidentified salts that decreased stain uptake, which is why the Biological Stain Commission increased the minimum dye content requirement for eosin to $90 \%$.

c) Stain is insufficiently concentrated. Increase the concentration. Dissolve in $70 \%$ ethanol.

d) Include $0.5 \mathrm{~mL}$ glacial acetic acid per $100 \mathrm{~mL}$ stain.

e) Stain longer. Do the equivalent of bracketing photographic exposures: double the staining time (e.g., 30 seconds, $1,2,4$ and 8 minutes) to quickly find the correct time. Very thin sections require longer staining times than do thicker sections, simply because there's less there.

5) If too much eosin is getting out, possible reasons include:

a) Inappropriate rinses. An alkaline rinse, for example, will quickly undo the staining.

b) Rinsing too long (unlikely).

c) Water in xylene. Check for water droplets in the xylene. Place a drop on a slide and examine with the substage condenser aperture diaphragm closed to exaggerate the appearance.

d) Mounting medium related. A few mounting media fade dyes substantially, even in the dark.

e) Oddball causes (e.g., xylene substitutes)

6) Other. Changing the fixative can sometimes cause problems.

A more intense eosin stain was made by Don Myers. He dissolved eosin, phloxine B, and Biebrich scarlet in $19 \%$ ethanol to make a modified eosin that stained tissues an intense red, with various shades. Now deceased, Don was Chief Histotechnologist in the Department of Pathology at The Johns Hopkins Hospital for many years. The histology lab is named in his memory, as I recall.

Don's formula is:
Biebrich scarlet (ws) (C.I. No. 26905) $0.4 \mathrm{~g}$

Eosin Y, (C.I. No. 45380$) 5.0 \mathrm{~g}$

Phloxine B (C.I. No. 45410) $2.1 \mathrm{~g}$

$95 \%$ ethanol $200 \mathrm{~mL}$

Distilled water $800 \mathrm{~mL}$

The weights given are for total dye content. Greater amounts, therefore, must actually be added since no available dyes have $100 \%$ dye content. The adjustment must be made for each lot of dye, using the dye content given for the lot used. Biebrich scarlet is not certified by the Biological Stain Commission; no percent dye content will be stamped on its label. Acetic acid is not added. Combine the ingredients at room temperature, stir, and filter before using. Staining time is a few dips.

Bob Richmond describes it as "an eye blasting eosin-mix" that "stained all kinds of stuff you can't see with the wimp eosin that's around nowadays." Bob is a Johns Hopkins-trained pathologist who used to look at tissues stained by this formula.
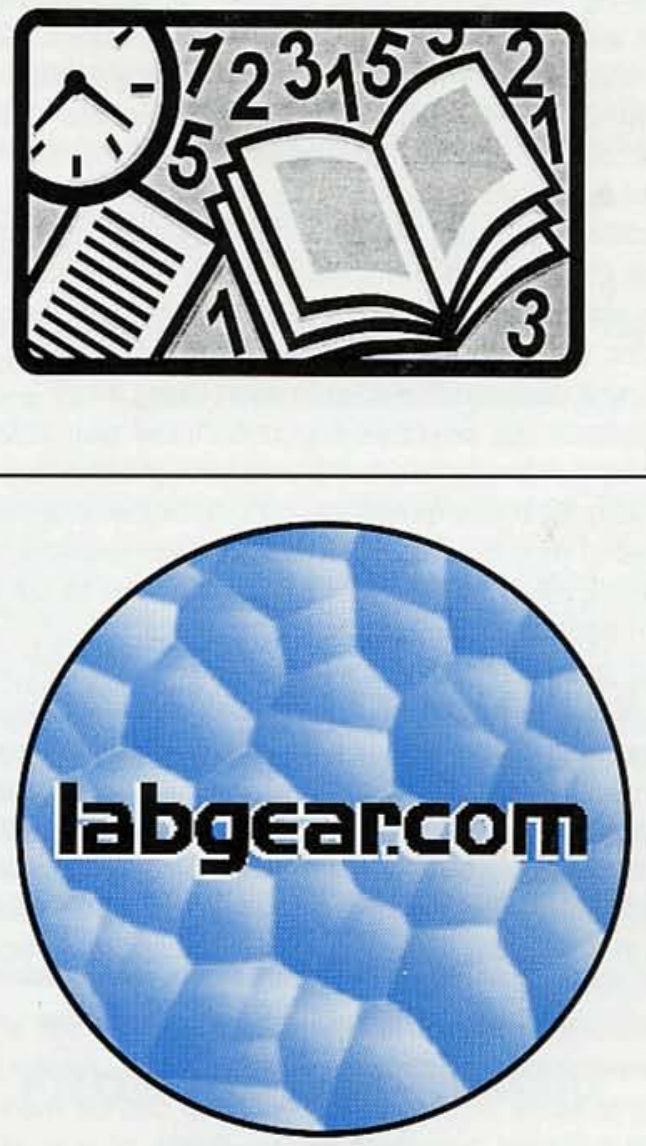

Problem: Find the right equipment, ... And find it now !! Solution: labgear.com

- Gather information about microscopy techniques

- Research and find analytical equipment

- Search by technique and then by manufacturer

- Video clips of laboratories and trade shows

- Calendar, new product features, tips from experts

20/20 Foresight Scientific Marketing 423/984-8683 